\title{
Bronchial and gastrointestinal cryptosporidiosis in AIDS
}

\author{
R.S. GOODSTEIN, DO \\ C.S. COLOMBO, DO \\ M.A. ILLFELDER, DO \\ R.E. SKAGGS, DO
}

\begin{abstract}
Cryptosporidiosis is a coccidial protozoan initially reported in domestic animals. It is primarily a gastrointestinal organism that does not invade mucosa. It was first described in 1976 in developing countries as an etiology of infantile diarrhea with inanition and malnutrition. Gastrointestinal involvement in patients with AIDS has been increasingly reported. We report a case of combined gastrointestinal and bronchial cryptosporidiosis. Cryptosporidium is an acid-fast organism which was successfully treated with erythromycin.
\end{abstract}

Cryptosporidiosis, a protozoan etiology for diarrhea and weight loss, is self-limiting in the immunocompetent host. However, in the immunocompromised host, such as an AIDS patient, it has a chronic, debilitating, inanitiating course.

We describe the progression of disseminated (gastrointestinal and bronchial systems) cryptosporidiosis in an AIDS patient and discuss the diagnosis and treatment of the organisms in such cases.

\section{Report of case}

A 27-year-old, homosexual, quadriplegic man was admitted on October 26, 1986, complaining of nausea, emesis, progressive fatigue, anorexia, hematochezia, abdominal bloating, and diarrhea of one week's duration. The patient had had a 55-pound weight loss over the previous four-month period. The past medical history included an eight-month history of a positive HIV-antibody titer and a four-month history of malabsorption syndrome. His quadriplegia was due to biopsy-proved CNS toxoplasmosis. On admission, his medications included desipramine, leucovorin, ketoconazole, metronidazole, and pyrimethamine.

Physical examination revealed an afebrile, normotensive, cachectic male with clear lung fields. The abdomen was distended. The patient had a regular cardiac rate and rhythm.

Laboratory studies revealed leukopenia (with $7 \%$ band neutrophils), anemia, hypoalbuminemia, and an elevated alkaline phosphatase value. On the third hospital day, colonoscopy with brushings and washings was performed, and, when stained, the washings demonstrated Cryptosporidium. Cultures of blood obtained on November 12 were positive for Streptococcus fecalis and Staphylococcus epidermidis.

During hospitalization, the patient complained of cough and was noted to have hemoptysis, and pulmonary consultation was obtained. The chest film suggested a vague left infrahilar infiltrate. Bronchoscopy performed on November 14 showed mucosal edema and mild erythema of the airways. Protected catheter brushings (Fig 1) and biopsy specimens (Fig 2) were obtained and were positive for Cryptosporidium on modified Kinouyn staining.

The patient was treated with erythromycin $(500 \mathrm{mg}$ four times daily), and the cough and diarrhea decreased and the hemoptysis resolved. Hyperalimentation via Hickman catheter was initiated. He experienced re-exacerbation of CNS toxoplasmosis and became encephalopathic. He died approximately eight weeks after admission, on January 17, 1987, of CNS toxoplasmosis, AIDS, and AIDS-wasting syndrome.

\section{Discussion}

As the clinical scope of AIDS expands in both diagnostic and therapeutic areas, it is understandable that the microbiologic yield likewise will increase. Cryptosporidium is, for the most part, a self-limiting, symptomatically limited, diarrheal illness.

In AIDS, however, it may become more invasive and potentially fatal because of the patient's cellular immunodeficiency. It may involve the entire gastrointestinal tract, including the pancreaticobiliary tree, as well as the respiratory system. ${ }^{1,2}$ Symptoms of cough and dyspnea in AIDS are more usually attributed to more commonly known pathogens, and, therefore, appropriate diagnostic staining techniques often are not considered. In fact, because Cryptosporidium is primarily a gastrointestinal organism, the diarrhea it causes usually is the clinician's main concern.

A coccidial protozoan with 11 species, Cryptosporidium initially was described in mice in $1907 .^{3}$ The organism has been found in cats, dogs, lambs, 


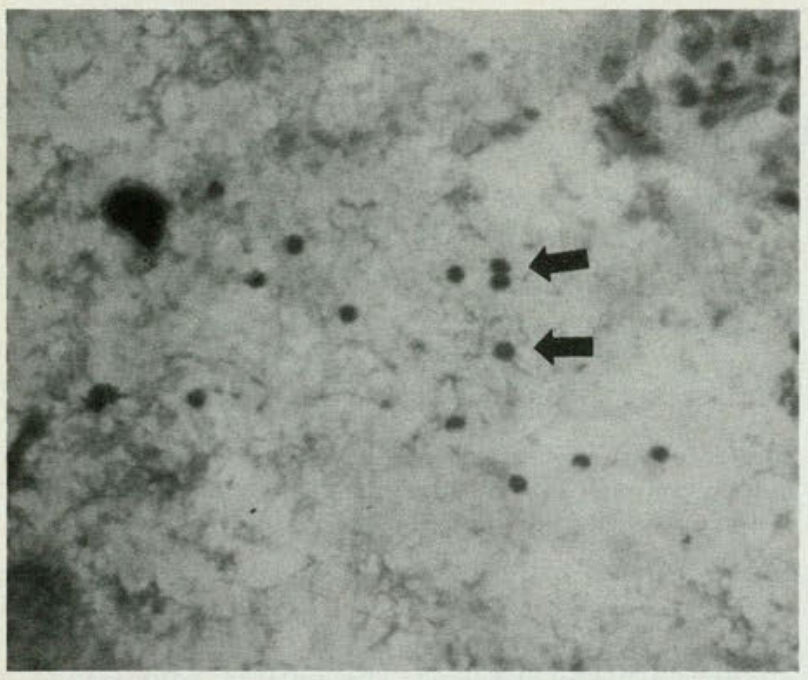

Fig 1. Protected catheter bronchial brushing demonstrates Cryptosporidium (arrows) (modified Kinouyn stain; original magnification, $\times 400$ ).

pigs, snakes, and turkeys ${ }^{4}$ with the highest carrier rate in domestic animals. It was first reported in humans in $1976 . .^{5}$

Gastrointestinal Cryptosporidium does not invade mucosa ${ }^{2}$; rather, trophozoites attach to the alimentary tract mucosal surface, a characteristic that favors diagnosis. Because bronchial Cryptosporidium also is not a mucosal invader, it lends itself to both bronchial lavage and biopsy, as were used for diagnosis in our patient. The organism's life cycle is completed extracellularly. It is transmitted via fecal-oral route, with a five- to 10-day incubation period. ${ }^{1}$

The organism has been found primarily in immunocompetent hosts, especially travelers to developing nations, animal handlers, and, most commonly, children in developing nations. ${ }^{6,7}$ Symptoms include, but are not limited to, colicky abdominal pain, nausea, watery and malodorous diarrhea, anorexia, emesis, weight loss, malaise, and fever. ${ }^{7}$

In the immunocompetent patient, symptoms are significantly shorter, with the average duration of diarrhea being approximately 10 to 21 days, while $50 \%$ of immunocompromised patients have diarrhea for longer than four months,${ }^{8}$ with a diarrheal fluid volume as large as $3 \mathrm{~L} /$ day. ${ }^{4}$

In our case, diagnosis was made in both colonic and bronchial mucosa with a modified Kinouyn stain. Oocysts can be identified in stool; $\mathrm{Ma}$ and Soave $^{9}$ noted that a three-step stool examination (iodine stain, modified Kinouyn stain, and sugar

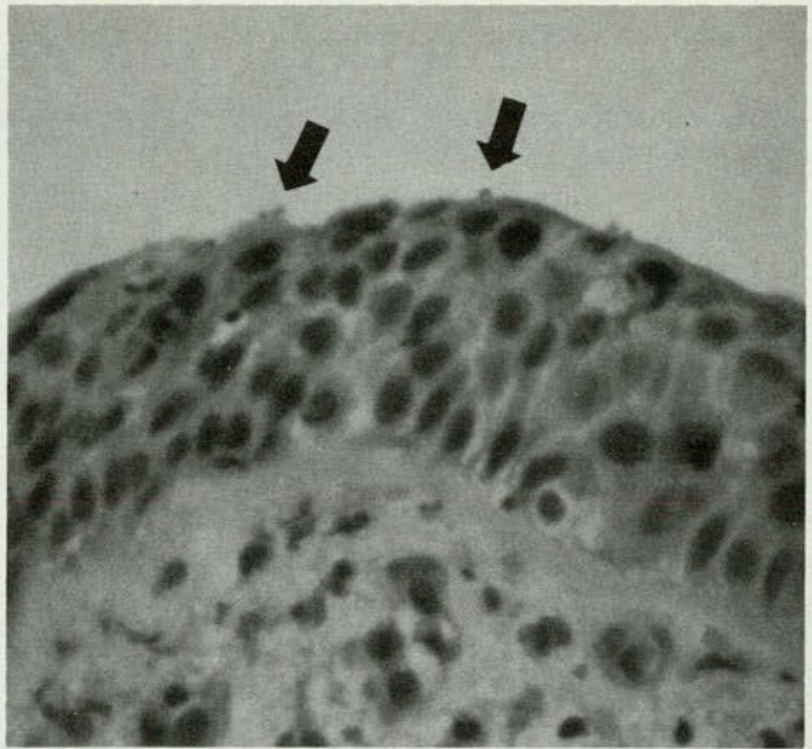

Fig 2. Bronchial biopsy speciment shows Cryptosporidium (arrows) (hematoxylin and eosin stain; original magnification, $x$ 250).

flotation) do not improve the sensitivity of the Kinouyn stain. Stools usually are negative for leukocytes and blood.

Anticryptosporidial antibodies can be identified within two weeks of the onset of symptoms and may have a role in humoral immunity. The presence of humoral immunocompetency correlates to duration and the degree of clinical symptoms. In children in developing nations, breast feeding with passive immunizations is protective. ${ }^{6}$

The organism produces no toxin. Watery diarrhea is produced by mucosal attachment of the Cryptosporidium trophozoite, and there is atrophy of the small intestinal villae and mononuclear infiltration. ${ }^{10}$ Mortality is higher than $50 \%$ when there are electrolyte disturbances, dehydration, inanition, malnutrition, and attending complications. For our patient, total peripheral hyperalimentation was used.

Current therapy is limited to macrolides antibiotics, including spiramycin $(1 \mathrm{~g}$, three or four times daily) and erythromycin (500 mg to $1,000 \mathrm{mg}$, three or four times daily), with symptomatic improvement noted in $50 \%$ of cases. ${ }^{11}$ In our patient, erythromycin resulted in a decrease of diarrhea and cough and resolution of hemoptysis. Because in the normal host the clinical course is so short-lived, therapy usually is reserved for the immunodeficient patient. 


\section{Summary}

Mortality and morbidity secondary to cryptosporidiosis reflect host immunocompetency or immunodeficiency. The immunodeficient host may have a protracted, voluminous diarrhea, which leads to dehydration, electrolyte disorders, and malnutrition, while the immunocompetent host will have self-limited disease.

As far as we can ascertain, Cryptosporidium pneumonitis has been associated only with cell-mediated immunodeficiency syndromes, such as AIDS. In these cases, erythromycin has been used with symptomatic improvement. No long-term studies on microbiologic cure are available. We suggest that erythromycin probably decreases the protozoan load, which reduces mucosal involvement to a point where symptoms resolve.

1. Gross TL, Wheat J, Barlett M, et al: AIDS and multiple system involvement with Cryptosporidium. Am J Gastroenterol 1986;81:456-458.

2. Forgacs $\mathrm{P}$, Tarshis $\mathrm{A}, \mathrm{Ma} \mathrm{P}$, et al: Intestinal and bronchial cryptosporidiosis in an immunodeficient homosexual male. Ann Intern Med 1983;99:793-794.
3. Tyzzer EE: A sporozoan found in the peptic glands of the common mouse. Proc Soc Exp Biol Med 1907;5:12-13.

4. Cryptosporidiosis; editorial. J Infec 1985;10:1-3.

5. Soave R, Ma P: Cryptosporidiosis: Traveler's diarrhea in two families. Arch Intern Med 1985;145:70-72.

6. Casemore DP, Sands RL, Curry A: Cryptosporidium species: A 'new' human pathogen. J Clin Pathol 1985;38:1321-1336.

7. Cooper DA, Wodak A, Marriot DJ, et al: Cryptosporidiosis in the acquired immune deficiency syndrome. Pathology 1984;16:455-457.

8. Navin TR, Juranek DD, Cryptosporidiosis: Clinical, epidemiological and parasitic review. Rev Infect Dis 1984:6:313-327.

9. Ma P, Soave R: Three-step stool examination of cryptosporidiosis in 10 homosexual men with protracted watery diarrhea. $J$ Infect Dis $1983 ; 147: 824-828$.

10. Berkowitz CD: AIDS and parasitic infections, including Pneumocystis carinii and cryptosporidiosis. Pediatr Clin North Am 1985;32:933952

11. Berkowitz CD, Seidel JS: Spontaneous resolution of cryptosporidiosis in a child with acquired immunodeficiency syndrome, letter. $\mathrm{Am}$ $J$ Dis Child 1985;137:967.

From the Department of Internal Medicine (Drs Goodstein, Colombo, and Illfelder) and Department of Pathology (Dr Skaggs), Memorial Hospital, York, Pa.

Reprint requests to Dr Goodstein, 1777 5th Ave, York, PA 17403. 


\section{The National Osteopathic Foundation Announces:}

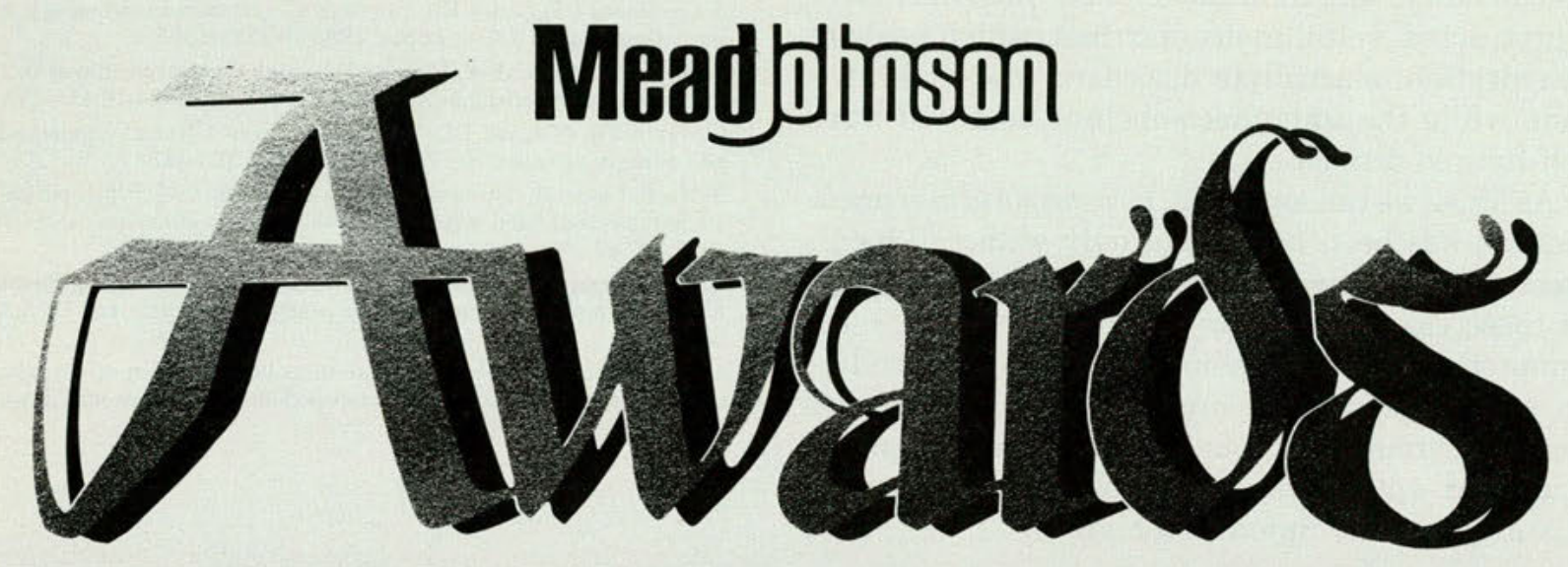

\section{FOR GRADUATE TRAINING}

Six $\$ 5,000$ awards in 1989 plus round trip transportation to AOA annual meeting

Award recipients will be the guests of honor at an awards

breakfast to be hosted by Mead Johnson at the AOA Annual Meeting

D For residency training in AOA approved specialties

D The National Osteopathic Foundation administers all funds and selection of recipients

The Mead Johnson Awards for Graduate Training in osteopathic medicine provide financial assistance to selected graduates toward completion of a year of residency training. Mead Johnson has participated by providing funds for this joint endeavor since 1956.

Grants are available to any osteopathic physician within four years of date of graduation from an osteopathic college. The recipient may choose any hospital approved for residency training by the American Osteopathic Association.

Application forms may be obtained by writing to:

Committee on Educational Grants

The National Osteopathic Foundation 142 E. Ontario, 2nd Floor

Chicago, Illinois 60611

(800) 621-1773 (Ext. 5850) 